\title{
Bariatric Surgical Services Within a Pandemic Can Continue Safely: the Initial Experience of a UK Centre of Excellence
}

\author{
Osama Moussa $^{1,2} \cdot$ Patricia Ortega $^{1} \cdot$ Sami Mansour ${ }^{1} \cdot$ Sara Flod $^{1}$ - Jonathan Cousins ${ }^{1} \cdot$ Saira Hameed ${ }^{1} \cdot \operatorname{Trisha~Tan~}^{1}$. \\ Alex Miras ${ }^{1}$ - Harvinder Chahal ${ }^{1}$. Sherif Hakky ${ }^{1} \cdot$ Krishna Moorthy $^{1,2} \cdot$ Christos Tsironis $^{1} \cdot$ Ahmed Ahmed $^{1,2}$. \\ Sanjay Purkayastha ${ }^{1,2}$
}

Received: 3 June 2021 / Revised: 9 July 2021 / Accepted: 14 July 2021 / Published online: 3 August 2021

(C) The Author(s), under exclusive licence to Springer Science+Business Media, LLC, part of Springer Nature 2021

Coronavirus disease 2019 (COVID-19) is a respiratory disease caused by the novel severe acute respiratory syndrome coronavirus 2 (SARS-CoV-2), which has reached pandemic status as declared by the World Health Organization (WHO) on 11 March 2020 [1]. The epidemic of obesity had progressed to global pandemic status, even before this recent viral pandemic [2]. The outbreak of COVID-19 has caused great concern among patients with obesity. COVID-19 may have detrimental effects on obesity, including more significant risks of severe complications due to increased medical comorbidities in this population [3].

The COVID-19 pandemic has posed challenges to the healthcare system in the UK [4]. Given the large and growing backlog of patients awaiting medically necessary elective surgery (not just cancer-related surgery but benign elective cases), there is now an urgent need to rebuild surgical activities across the nation [5]. How to safely restart surgical programs [6] in the context of ongoing community spread and localised outbreaks of COVID-19 remains unclear.

The authors describe an initial experience of a tertiary bariatric unit restarting promptly after the recommencement of surgery within the UK using an already established local prospectively collated database cross-referenced with the National Bariatric Surgical Registry (NBSR). The details of the demographic, operative and clinical data were interrogated by two authors (OM, PO) for validity.

Sanjay Purkayastha

s.purkayastha@imperial.ac.uk

1 Department of Surgery and Cancer, Imperial College, London W2 $1 \mathrm{NY}, \mathrm{UK}$

2 Imperial Weight Centre, St Mary's Hospital, Imperial College NHS Healthcare Trust, 10th Floor QEQM building, Praed Street, London W2 1NY, UK
All cases were performed electively within an established weight management unit. All patients on the waiting list were prioritised except those requiring a prolonged or complicated postoperative stay. All high-risk patients (age $>60$ years and patients with diabetes or with underlying lung pathology) were further prioritised to a priority 2 (to be done within 4 weeks) [7]. This was confirmed by the Tier 4 multidisciplinary team (MDT) meeting using the Edmonton Obesity Staging System [8] and clinical harm scores assigned as per the Royal College of Surgeons (RCS) England guidelines [9].

An agreed COVID-19 safe pathway was devised, a set protocol for standardisation after discussion at the MDT meeting. After completing remote consultations, patients were advised to self-isolate before surgery for 1 week. Three days before surgery, an in-person surgical pre-assessment was performed, including an additional COVID-19 PCR test. All surgeries were undertaken on an elective pathway and screened for COVID-19 symptoms and a routine temperature check, and their COVID-19 PCR test was repeated. All surgeries were performed in a specialist laparoscopic operating theatre. Surgical wounds were closed with absorbable sutures to avoid returning to the hospital for clip removal. Postoperatively, patients were supported in a specialist three-bed-isolated bariatric ward. All staff were tested weekly with COVID-19 PCR tests. Symptomatic staff were excluded from the site and referred to primary care. In addition, anaesthetic pathways were in place at the time of surgery, fully compliant with current guidance from the Royal College of Anaesthesia, specifically with aerosol-generating procedures [10].

Overall, 204 patients were contacted, of which 180 $(88.2 \%)$ underwent surgery. Twenty $(9.8 \%)$ patients declined surgery, and $2(0.98 \%)$ patients opted to have surgery abroad, and $2(0.98 \%)$ patients did not respond. One hundred and eighty patients underwent bariatric procedures from July 2020 to early December 2020 when bariatric services 
were suspended again due to the second UK wave of COVID19. The number of operations performed was, in fact, slightly increased during the pandemic period (170 in 2019 vs 180 in 2020) despite the reformed pathways.

The majority of patients were female $(76.8 \%)$, and the mean age was 44.5 (11.8) years. Hypertension (50.3\%), dyslipidaemia (43.6\%) and diabetes $(34.3 \%)$ were the most prevalent obesity-associated comorbidities. Functional mobility was documented in $75.7 \%$ of patients, and the most prevalent functional status was tolerating at least two flights of stairs $(51.4 \%)$. The majority of patients were of the American Society of Anaesthesiology (ASA) grade 3 (47.5\%), followed by grade $2(40.9 \%)$.

Baseline descriptive measurements were verified at the date of the Tier 4 multi-disciplinary meeting discussion. The average time from Tier 4 MDT to surgery was 53.5 (SD 24.6) weeks. The mean weight immediately pre-operatively on the day of surgery was recorded in 137 cases $(76.1 \%)$ and was 122.0 (SD 27.5) $\mathrm{kg}$, and on the first postoperative day ten follow-up was 115.9 (SD 39.1) $\mathrm{kg}$.

All procedures were carried out laparoscopically. The most frequently performed procedure was sleeve gastrectomy (42\%), followed by Roux-en-Y gastric bypass (30.4\%). Procedures were primary in $90.1 \%$ vs $9.9 \%$ revisional. The primary performing surgeon was a fellow $(39.8 \%)$, consultant $(37.6 \%)$ and training registrar $(22.1 \%)$. The majority of procedures $(97.2 \%)$ were performed electively. Patients with type 2 diabetes $(46.8 \%)$ were more likely to have Roux-en-Y gastric bypass. Patients with hypertension $(46.2 \%)$ or dyslipidaemia $(46.8 \%)$ were more likely to have a sleeve gastrectomy.

The mean postoperative length of stay was 2.2 (SD 0.9) days. The first nurse specialist remote review took place 9.5 days (SD 3.1) post-op. Overall, there were 6 (3.3\%) (ClavienDindo grade II) complications and $2(1.1 \%)$ Clavien-Dindo IIIb complications. There were 7 (4.1\%) postoperative morbidities, of which 2 were chest infections, a wound infection, arrhythmia, and $2(1.1 \%)$ needed a second procedure within 3 months of the index surgery (one for small bowel obstruction due to a paraumbilical hernia and one due to postoperative staple line bleed). There were no mortalities amongst our cohort, and there was only one COVID-19-related diagnosis identified amongst the whole cohort re-presenting after 14 days of discharge, with no adverse clinical sequelae other than the admission.

Overall, UK bariatric services have been markedly reduced or totally cancelled throughout the pandemic. Currently, at the time of writing (February 2021), bariatric surgical services in most UK hospitals continue to be suspended. There is a clinical drive to restart services before the pandemic completely settles, demonstrated by nationwide-based surveys [6].

The authors have reported a pathway based on global and national recommendations applied in a multi-disciplinary manner. This involved a bariatric MDT working together to formulate new pathways using the latest and often changing evidence base and seeking management and board approval. Once a safe local protocol was established, this became a routine to which all clinicians ensured continuity. The authors demonstrate a considerable number of bariatric surgeries carried out in a brief period compared with other UK institutions; this was also cross-checked with the NBSR. Both local and national database was maintained in parallel for quality control and to aid retrospective audit analysis. This data also demonstrates that consultant-led care allows the primary operating surgeon to be a trainee (fellow and registrar), indicating that specialist training opportunities can continue in a pandemic with appropriate planning.

This experience also demonstrates that reinitiating an MDT-facilitated, COVID-19 risk-managed pathway bariatric surgery (in a cohort of patients who already have a higher risk of COVID-related morbidity) is both feasible and safe within an ongoing pandemic. Overall, this is the most extensive COVID-19 bariatric case series in the UK and describes the feasibility of a high bariatric caseload and safety in a specialised tertiary bariatric unit.

Acknowledgements The authors would like to acknowledge the work and collaboration of all of the bariatric MDT members in the department who have worked so tirelessly during the pandemic to construct and deliver the whole patient pathway to continue our service safely with excellent patient feedback and outcomes.

\section{Declarations}

Ethics Approval For this type of study, formal consent is not required.

Consent to Participate Informed consent does not apply.

Conflict of Interest The authors declare no competing interests.

\section{References}

1. World Health Organization (WHO). Coronavirus disease 2019 Situation Report - 5212 March 2020. World Health Organ. 2020;2633. Available from: https://www.who.int/emergencies/ diseases/novel-coronavirus-2019. Accessed 30 Mar 2021

2. Roth J, Qiang X, Marbán SL, Redelt H, Lowell BC. The obesity pandemic: where have we been and where are we going? Vol. 12 Suppl 2, Obesity research. 2004.

3. Sockalingam S, Leung SE, Cassin SE. The impact of COVID-19 on bariatric surgery: re-defining psychosocial care. Obesity. 2020;

4. Berlin G, Bueno D, Gibler K SJ. Cutting through the COVID-19 surgical backlog. McKinsey Co. 2020.

5. Nepogodiev D, Bhangu A. Elective surgery cancellations due to the COVID-19 pandemic: global predictive modelling to inform surgical recovery plans. Br J Surg [Internet]. 2020; Available from: https://doi.org/10.1002/bjs.11746

6. Moussa O, Zakeri R, Arhi C, et al. Impact of COVID-19 on obesity management services in the United Kingdom (The COMS-UK 
study). Obes Surg. 2020; Available from; https://doi.org/10.1007/ s11695-020-05005-1.

7. Surgeons RC of. Clinical guide to surgical prioritisation during the coronavirus pandemic. 2021

8. Sharma AM, Kushner RF. A proposed clinical staging system for obesity. Int J Obes. 2009;33(3):289-95.

9. The Federation of Speciality Surgical Association. Clinical guide to surgical prioritisation during the coronavirus pandemic $\mathrm{T}$
10. Sciences M, Nhs T, Units PC, Rooms R. Anaesthesia and critical care: guidance for clinical directors on preparations for a possible second surge in COVID-19. ICM Anaesth COVID-19 [Internet]. 2020;1-11. Available from: https://icmanaesthesiacovid-19.org/s/ Second-Surge-Guidance.pdf. Accessed 21 Sept 2020.

Publisher's Note Springer Nature remains neutral with regard to jurisdictional claims in published maps and institutional affiliations. 